\title{
Effect of Intra-Articular Injection of Triamcinolone Acetonide for Wrist, Elbow and Shoulder Pain in Patients with Rheumatoid Arthritis: Retrospective Study
}

\author{
Akihiro Fukui ${ }^{1, ~ *, ~ T a k a s h i ~ Y o s h i i ~}{ }^{2}$, Hideki Yamada ${ }^{3}$ \\ ${ }^{1}$ Department of Orthopedic Surgery, Nishinokyo Hospital, Nara, Japan \\ ${ }^{2}$ Department of Orthopedic Surgery, Saiseikai Cyuwa Hospital, Nara, Japan \\ ${ }^{3}$ Department of Internal Medicine, Nijyo-Ekimae Clinic, Nara, Japan \\ Email address: \\ ma77vv77ml@kcn.jp (A. Fukui) \\ ${ }^{*}$ Corresponding author
}

\section{To cite this article:}

Akihiro Fukui, Takashi Yoshii, Hideki Yamada. Effect of Intra-Articular Injection of Triamcinolone Acetonide for Wrist, Elbow and Shoulder Pain in Patients with Rheumatoid Arthritis: Retrospective Study. Journal of Surgery. Vol. 7, No. 6, 2019, pp. 168-179. doi: $10.11648 /$ j.js.20190706.14

Received: October 5, 2019; Accepted: November 5, 2019; Published: November 8, 2019

\begin{abstract}
Background: Many rheumatoid arthritis patients with joint pain refuse the indicated synovectomy, joint arthroplasty, or similar surgical procedures, opting instead to receive intra-articular injections of corticosteroid. Methodology: We evaluated the clinical benefit and safety of intra-articular injections of triamcinolone acetonide in 153 (132 females and 21 men) rheumatoid arthritis patients with wrist, elbow and shoulder pain by analyzing the number of injections, decrease in pain measured on a visual analog scale (VAS), changes in carpal height ratio (CHR), radio carpal distance ratio (RCDR) and radial rotation angle (RRA) on X-ray imaging, and adverse effects in the subcutaneous tissue and extensor tendons. Results: Over the 8 -year study period (average 6 yrs.), the mean numbers of intra-articular triamcinolone acetonide injections per patient were 3.7 for 180 wrists in 118 patients, 2.2 for 45 elbows in 36 patients, 2.8 for 60 shoulders in 44 patients. Mean improvements in VAS pain scores from baseline were as follows: wrist, from 75 to $11 \mathrm{~mm}$; elbow, from 79 to $17 \mathrm{~mm}$; shoulder, from 54 to $11 \mathrm{~mm}$. The group that received the injections showed no significant changes in CHR or RCDR, whereas RRA changed significantly decrease $(\mathrm{P}<0.05)$. In the group that did not receive intra-articular injections, no significant changes were observed in CHR, RCDR and RRA over a mean follow-up period of 8 years. No abnormalities were observed in subcutaneous tissue. Conclusions: Overall, $>90 \%$ of the patients of all disease grades responded to an average of 1-4 intra-articular triamcinolone acetonide injections, which were effective for pain relief in the wrist, elbow and shoulder joints over both the short and long term. Skin atrophy or extensor tendon rupture due to injection did not occur.
\end{abstract}

Keywords: Rheumatoid Arthritis, Triamcinolone Acetonide, Joint Pain, Larsen Scoring System, Biologic

\section{Introduction}

Synovitis is commonly associated with the progressive deterioration of wrist, elbow and shoulder joints in patients with rheumatoid arthritis (RA). Synovectomy, joint arthroplasty, or a similar surgical procedure is usually indicated for joint pain in these patients; however, many refuse such surgery and opting instead to receive intra-articular injections of corticosteroid. In such cases, we administer an injection of $20 \mathrm{mg}$ of triamcinolone acetonide and $5 \mathrm{~mL}$ of $1 \%$ lidocaine hydrochloride into the unilateral wrist, elbow and shoulder. Patients with shoulder pain receive an additional $25 \mathrm{mg}$ of hyaluronic acid injected into the shoulder joint. This study evaluated the clinical benefit and safety of these intra-articular injections of triamcinolone acetonide and analyzed the number of injections according to the Larsen scoring system grade; the number of injections with which a biologic was used or not used; the decrease in pain, measured using a visual analog scale (VAS); changes in carpal height ratio (CHR), radio carpal distance ratio (RCDR) and radial rotation angle (RRA) on dorso-palmar plain X-ray imaging; and any adverse effects of the injections, including 
on subcutaneous tissue and the extensor tendons.

\section{Materials and Methods}

\subsection{Methodology}

The study included patients with RA who experienced wrist elbow and shoulder joint pain but refused surgery from November 2009 to December 2017. All received unilateral injections of triamcinolone acetonide $(20 \mathrm{mg})$ and $1 \%$ lidocaine hydrochloride $(5 \mathrm{ml})$ into the joint using a 23-guage needle. Patients with shoulder pain received an additional 25 $\mathrm{mg}$ of hyaluronic acid injected into the shoulder joint. Only patients available for at least 6 months of follow-up after the first injection were included in the study. The patients that achieved sustained pain relief after an injection were given a subsequent injection only when the pain recurred. Patients who did not respond to the injection were given a second injection 2 weeks later. Those who did not respond to the second injection were discontinued from treatment and encouraged to undergo surgery. At the beginning of the injection, an attempt was made for pain in the wrist joint. However, there were many cases in which the patient complained of elbow and shoulder pain, and injection was also attempted in each joint.

\subsection{Number of Injections}

Overall, 153 patients (132 females and 21 men) with a mean duration of RA of 10.9 (2-22) years, mean age at the time of the first injection of 64 (27-85) years, and mean follow-up period of 32 months ( 6 months to 8 years and 1 month) were enrolled. No patient had complication of diabetes mellitus or glaucoma. Tables 1 to 5 were created by classifying grades based on the X-ray imaging of the wrists, then elbow and shoulder joints were added.

The 180 wrists (118 patients) were graded on the Larsen scoring system [1] as follows: grade I, 48 wrists (32 patients); grade II, 46 wrists (30 patients); grade III, 51 wrists (33 patients); and grade IV, 35 wrists (23 patients). The 45 elbows (36 patients) were graded as follows: grade I, 24 elbows (17 patients); grade II, 7 elbows ( 7 patients); grade III, 9 elbows (8 patients); and grade IV, 5 elbows (4 patients). The 60 shoulders (44 patients) were graded as follows: grade I, 37 shoulders (25 patients); grade II, 2 shoulders (2 patients); grade III, 7 shoulders (6 patients); and grade IV, 14 shoulders (11 patients). Since joint injection was started from the wrist joint, it was created mainly with grades I to IV of the Larsen classification of the wrist. Therefore, Table 1 is centered on grade I of the wrist, Table 2 is centered on grade II, Table 3 is centered on grade III, Table 4 is centered on grade IV. Table 5 is the part of the case where the injection was made to the elbow and shoulder joints, then each grade and the number of injections were listed.

\subsection{Use of Biologics}

Of the 49 patients treated with a biologic, 23 received etanercept (grade I, 4; grade II, 7; grade III, 7; and grade IV, 5); 14 received infliximab (grade I, 2; grade II, 3; grade III, 4; and grade IV , 6), 9 received adalimumab (grade I, 1; grade II, 2; grade III, 4; and grade IV , 2), 8 received abatacept (grade 1, 1; grade II, 2; grade III, 1; and grade IV, 4), 6 received tocilizumab (grade I, 1; grade II, 1; grade III, 3; and grade IV, 1), 3 received golimumab (grade I, 2; and grade IV, 1), and 1 (grade III) received certolizumab. Some of the patients received $>1$ biologic.

\subsection{Decrease in Pain}

The following endpoints were recorded for each patient: the number of injections and grade of the joint; the numbers of injections with which a biologic was used or not used; the decrease in pain, as measured on a Visual Analogue Scale (0100); VAS surveyed 1 month after intra-articular triamcinolone acetonide injection.

\subsection{Adverse Effects of Intra-articular Triamcinolone Acetonide Injections}

Any adverse effects on the subcutaneous tissue and extensor tendons of triamcinolone acetonide injection into the joints were checked.

\subsection{Changes in CHS, RCDR, and RRA with and Without Injections of Triamcinolone Acetonide}

In addition, we analyzed 2 groups of patients with advanced wrist joint destruction (Larsen grades III and IV, indicating moderate and severe joint destruction, respectively). The first comprised 37 patients (grade III, 22; grade IV, 15) who received injections of $20 \mathrm{mg}$ of triamcinolone acetonide and 5 $\mathrm{mL}$ of $1 \%$ lidocaine hydrochloride. The second comprised 33 patients (grade III, 16; grade IV, 17) who did not receive these injections. For both groups, we examined differences in plain wrist X-ray images obtained at baseline and on the final day of the observation period, measuring changes in CHR, RCDR, and RRA according to the method of Youm [2] (Figure 1). $\mathrm{CHR}$, calculated from dorso-palmar X-ray imaging as the distance between the distal articular surfaces of the capitate of the third metacarpal to distal articular surface of the radius divided by length of third metacarpal, was used as an indicator of the degree of carpal compression $(\mathrm{a} / \mathrm{b})$. RCDR, calculated as the distance between the rotational center and a straight line passing through the radial side of the radius parallel to the length of the radius, was used as an indicator of carpal ulnar deviation. RRA (c/a), the angle between the line radially tangential to the shaft of the third metacarpal and the line tangential to the distal articular surface of the radius, was determined as an indicator of the radial rotation of the carpal bones $(\alpha)$. Student's t test was used for the statistical analyses. 


\begin{tabular}{|c|c|c|c|c|c|c|c|c|c|c|}
\hline \multirow[b]{2}{*}{ No. } & \multirow[b]{2}{*}{ age } & \multirow[b]{2}{*}{ sex } & \multirow[b]{2}{*}{ Bio. } & \multirow[b]{2}{*}{ site } & \multicolumn{2}{|c|}{ Wrist } & \multicolumn{2}{|c|}{ Elbow } & \multicolumn{2}{|c|}{ Shoulder } \\
\hline & & & & & grade & $\begin{array}{c}\text { injection } \\
\text { time }\end{array}$ & grade & $\begin{array}{c}\text { injection } \\
\text { time }\end{array}$ & grade & $\begin{array}{c}\text { injection } \\
\text { time }\end{array}$ \\
\hline 1 & 64 & $F$ & & $\mathrm{~L}$ & I & 3 & & & & \\
\hline 2 & 46 & $F$ & & L & I & 1 & & & & \\
\hline 3 & 76 & $F$ & & $\mathbf{R}$ & I & 1 & & & & \\
\hline 4 & 36 & $\mathrm{~F}$ & & $\mathbf{R}$ & I & 1 & & & & \\
\hline 5 & 65 & $F$ & & L & I & 1 & & & & \\
\hline 6 & 60 & $F$ & & L & I & 1 & & & & \\
\hline 7 & 54 & $F$ & & $\mathbf{R}$ & I & 1 & & & & \\
\hline 8 & 42 & $\mathrm{~F}$ & & $\mathbf{R}$ & I & 1 & & & & \\
\hline 9 & 80 & $\mathrm{~F}$ & & L & I & 1 & & & I & 1 \\
\hline 10 & 69 & $\mathrm{~F}$ & & $\mathbf{R}$ & I & 3 & & & & \\
\hline 11 & 64 & $\mathrm{~F}$ & & $\mathbf{R}$ & I & 3 & & & & \\
\hline 12 & 41 & $\mathrm{~F}$ & & L & $\mathrm{I}$ & 2 & & & & \\
\hline 13 & 78 & $\mathrm{~F}$ & Etanercept & $R$ & 1 & 1 & & & & \\
\hline \multirow{2}{*}{14} & \multirow{2}{*}{51} & \multirow{2}{*}{$\mathrm{F}$} & & $R$ & I & 1 & & & & \\
\hline & & & & L & I & 2 & & & & \\
\hline \multirow{2}{*}{15} & \multirow{2}{*}{61} & \multirow{2}{*}{$F$} & & $\mathrm{R}$ & I & 14 & & & & \\
\hline & & & & L & I & 11 & & & & \\
\hline \multirow{2}{*}{16} & \multirow{2}{*}{72} & \multirow{2}{*}{$F$} & & $\mathbf{R}$ & I & 5 & & & & \\
\hline & & & & L & I & 3 & & & & \\
\hline 17 & 83 & $F$ & & $\mathbf{R}$ & I & 6 & & & & \\
\hline & & & & L & I & 6 & & & & \\
\hline 18 & 47 & $\mathrm{~F}$ & & $R$ & I & 5 & & & & \\
\hline & & & & $\mathrm{L}$ & I & 3 & & & & \\
\hline 19 & 60 & $\mathrm{~F}$ & & $\mathrm{R}$ & I & 1 & & & & \\
\hline 10 & 00 & 1 & & L & I & 1 & & & & \\
\hline 20 & 59 & $F$ & & $\mathrm{R}$ & I & 4 & & & & \\
\hline 20 & 80 & & & L & I & 2 & & & & \\
\hline 21 & 64 & $\mathrm{~F}$ & & $R$ & I & 2 & & & I & 2 \\
\hline & & & & L & I & 3 & & & & \\
\hline 22 & 57 & $\mathrm{~F}$ & & $\mathrm{R}$ & I & 2 & & & & \\
\hline & & & & L & II & 2 & & & & \\
\hline 23 & 66 & $\mathrm{~F}$ & Etanercent & $\mathrm{R}$ & & & & & & \\
\hline & & & & L & I & 3 & & & & \\
\hline 24 & 43 & $F$ & Tofacitinib & $\mathrm{R}$ & I & 5 & I & 1 & I & 2 \\
\hline & & & 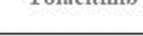 & L & I & 6 & & & I & 1 \\
\hline 25 & 42 & $\mathrm{~F}$ & Infliximab & $R$ & $\mathrm{I}$ & 7 & & & $\mathrm{I}$ & 3 \\
\hline & & & & L & I & 7 & & & I & 2 \\
\hline 26 & 50 & $F$ & Adlimumab & $R$ & I & 2 & & & & \\
\hline 20 & 50 & & Асаншаша & L & I & 2 & & & & \\
\hline 27 & 77 & 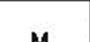 & Colinumel & $R$ & I & 3 & I & 1 & I & 2 \\
\hline $2 r$ & $m$ & $m$ & Goumumañ & L & I & 2 & I & 1 & I & 1 \\
\hline 28 & 69 & $F_{F}$ & Ftanercent & $R$ & I & 3 & III & 2 & & \\
\hline 20 & 09 & $r$ & Etanercept & L & I & 2 & III & 3 & & \\
\hline 29 & 78 & $\mathrm{~F}$ & Ahatacent & $R$ & I & 5 & & & & \\
\hline 29 & 10 & & & L & I & 7 & & & & \\
\hline 30 & 48 & $\mathrm{~F}$ & Golimumab & $\mathrm{R}$ & I & 4 & & & I & 1 \\
\hline & & & & L & I & 6 & & & I & 1 \\
\hline 31 & 65 & 4 & Ftongurent & $\mathrm{R}$ & & & & & I & 1 \\
\hline 31 & 05 & $m$ & Etanercept & $\mathrm{L}$ & I & 2 & & & I & 1 \\
\hline 32 & 40 & $F$ & Infliximab & $\mathbf{R}$ & $\mathrm{I}$ & 7 & & & I & 2 \\
\hline 02 & 40 & 1 & 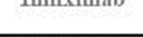 & L & I & 1 & & & & \\
\hline
\end{tabular}

Figure 1. Number of injections in grade I patients (based on the wrist). Thirty-two out of 118 patients were Grade I. 


\begin{tabular}{|c|c|c|c|c|c|c|c|c|c|c|}
\hline \multirow{2}{*}{ No. } & \multirow{2}{*}{ age } & \multirow{2}{*}{ sex } & \multirow{2}{*}{ Bio. } & \multirow{2}{*}{ site } & \multicolumn{2}{|c|}{ Wrist } & \multicolumn{2}{|c|}{ Elbow } & \multicolumn{2}{|c|}{ Shoulder } \\
\hline & & & & & grade & $\begin{array}{l}\text { injection } \\
\text { time }\end{array}$ & grade & $\begin{array}{l}\text { injection } \\
\text { time }\end{array}$ & grade & $\begin{array}{l}\text { injection } \\
\text { time }\end{array}$ \\
\hline 1 & 72 & $\mathrm{~F}$ & & $\mathrm{~L}$ & II & 1 & & & & \\
\hline 2 & 72 & $\mathrm{~F}$ & & L & II & 1 & & & & \\
\hline 3 & 70 & $\mathrm{~F}$ & & $\mathrm{~L}$ & II & 5 & & & & \\
\hline 4 & 71 & $\mathrm{~F}$ & & L & II & 1 & & & & \\
\hline 5 & 37 & $\mathrm{~F}$ & & $R$ & II & 2 & & & & \\
\hline 6 & 92 & M & & $R$ & II & 1 & & & & \\
\hline 7 & 60 & $\mathrm{~F}$ & & $R$ & II & 2 & & & & \\
\hline 8 & 59 & $\mathrm{~F}$ & & L & II & 2 & & & & \\
\hline 9 & 55 & $\mathrm{~F}$ & 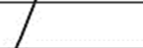 & $\mathrm{L}$ & II & 1 & & & & \\
\hline 10 & 72 & $\mathrm{~F}$ & & $\mathrm{~L}$ & II & 1 & III & 5 & II & 1 \\
\hline 11 & 66 & $\mathrm{~F}$ & & $R$ & II & 8 & II & 1 & & \\
\hline 12 & 71 & $\mathrm{~F}$ & Abatacept & $R$ & II & 1 & & & & \\
\hline 13 & 66 & M & Infliximab & L & II & 2 & & & I & 2 \\
\hline 14 & 60 & $\mathrm{~F}$ & Abatacept & $\mathrm{L}$ & II & 2 & & & & \\
\hline \multirow{2}{*}{15} & \multirow{2}{*}{65} & \multirow[b]{2}{*}{ M } & & $R$ & II & 1 & & & & \\
\hline & & & & L & II & 1 & & & & \\
\hline \multirow{2}{*}{16} & \multirow{2}{*}{64} & \multirow{2}{*}{$\mathrm{F}$} & & $R$ & II & 8 & & & I & 2 \\
\hline & & & & $\mathrm{L}$ & II & 6 & & & I & 1 \\
\hline \multirow{2}{*}{17} & \multirow{2}{*}{58} & \multirow{2}{*}{ M } & & $\mathrm{R}$ & II & 6 & I & 1 & & \\
\hline & & & & $\mathrm{L}$ & II & 5 & I & 1 & & \\
\hline \multirow{2}{*}{18} & \multirow{2}{*}{72} & \multirow{2}{*}{$\mathrm{F}$} & & $R$ & II & 4 & & & & \\
\hline & & & & $\mathrm{L}$ & II & 2 & & & & \\
\hline 19 & 61 & $F_{1}$ & & $R$ & II & 1 & & & & \\
\hline & & & & $\mathrm{L}$ & II & 1 & & & & \\
\hline 20 & 60 & $F$ & & $R$ & II & 1 & & & & \\
\hline 20 & 60 & $F$ & & $\mathrm{~L}$ & II & 1 & & & & \\
\hline 21 & 69 & $\mathrm{~F}$ & & $R$ & II & 2 & & & & \\
\hline 21 & 69 & $F$ & & $\mathrm{~L}$ & III & 1 & & & & \\
\hline 22 & 46 & $\mathrm{~F}$ & & $\mathrm{R}$ & II & 10 & I & 7 & & \\
\hline & & & & $\mathrm{L}$ & II & 11 & I & 1 & & \\
\hline 23 & 65 & 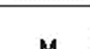 & & $\mathrm{R}$ & II & 16 & & & & \\
\hline 23 & & $M$ & & $\mathrm{~L}$ & II & 16 & & & & \\
\hline 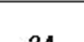 & 56 & 5 & Tton & $R$ & II & 1 & & & & \\
\hline 24 & 50 & $r$ & Etanercep & $\mathrm{L}$ & II & 1 & & & & \\
\hline 25 & 75 & F & Ftanercent & $\mathrm{R}$ & II & 3 & & & & \\
\hline & & & & $\mathrm{L}$ & II & 10 & & & & \\
\hline 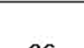 & $\mathrm{cos}^{-}$ & - & $\Gamma$ & $\mathrm{R}$ & II & 13 & & & & \\
\hline 26 & 62 & $F$ & Etanercept & L & II & 16 & & & & \\
\hline 27 & 87 & $\mathrm{~F}$ & Ftanercent & $\mathrm{R}$ & II & 1 & & & & \\
\hline & & & Etanercept & $\mathrm{L}$ & II & 7 & & & & \\
\hline 28 & 59 & $E$ & Tocilizung & $\mathrm{R}$ & II & 1 & & & & \\
\hline 28 & 59 & $F$ & 1ocilizumab & $\mathrm{L}$ & II & 2 & & & & \\
\hline 29 & 75 & $F$ & Ftanercent & $\mathrm{R}$ & II & 4 & & & & \\
\hline & & & & $\mathrm{L}$ & II & 4 & & & & \\
\hline 30 & 81 & $\mathrm{~F}$ & Etanercent & $R$ & II & 3 & & & & \\
\hline 30 & 81 & $\mathrm{~F}$ & Etanercept & $\mathrm{L}$ & II & 2 & & & & \\
\hline
\end{tabular}

Figure 2. Number of injections in grade II patients. Thirty out of 118 patients were Grade II. 


\begin{tabular}{|c|c|c|c|c|c|c|c|c|c|c|}
\hline \multirow{2}{*}{ No. } & \multirow{2}{*}{ age } & \multirow{2}{*}{$\operatorname{sex}$} & \multirow{2}{*}{ Bio. } & \multirow{2}{*}{ site } & \multicolumn{2}{|c|}{ Wrist } & \multicolumn{2}{|c|}{ Elbow } & \multicolumn{2}{|c|}{ Shoulder } \\
\hline & & & & & grade & 合計 & grade & $\begin{array}{c}\text { injection } \\
\text { time }\end{array}$ & grade & \begin{tabular}{|c|} 
injection \\
time
\end{tabular} \\
\hline 1 & 67 & $\mathrm{~F}$ & & L & III & 1 & III & 1 & I & 1 \\
\hline 2 & 59 & $\mathrm{~F}$ & & $\mathrm{~L}$ & III & 1 & & & & \\
\hline 3 & 62 & $M$ & & $\mathrm{R}$ & III & 1 & & & III & 1 \\
\hline 4 & 59 & $\mathrm{~F}$ & & $R$ & IIII & 2 & & & & \\
\hline 5 & 37 & $M$ & & $\mathbf{R}$ & III & 3 & & & & \\
\hline 6 & 54 & $M$ & & $\mathrm{~L}$ & III & 6 & & & & \\
\hline 7 & 54 & $\mathrm{~F}$ & & $\mathrm{~L}$ & III & 2 & & & & \\
\hline 8 & 57 & $\mathrm{~F}$ & Infliximab & L & III & 3 & & & & \\
\hline 9 & 84 & $\mathrm{~F}$ & Certolizumab & $R$ & III & 2 & & & & \\
\hline 10 & 65 & $\mathrm{~F}$ & Etanercept & $\mathbf{R}$ & III & 1 & & & & \\
\hline 11 & 71 & $\mathrm{~F}$ & Etanercept & $\mathbf{R}$ & III & 1 & & & & \\
\hline 12 & 77 & $\mathrm{~F}$ & $\begin{array}{l}\text { Etanercept, } \\
\text { Tocilizumab }\end{array}$ & $\mathbf{R}$ & III & 1 & & & & \\
\hline \multirow{2}{*}{13} & \multirow{2}{*}{75} & \multirow{2}{*}{$\mathrm{F}$} & & $\mathbf{R}$ & III & 2 & & & & \\
\hline & & & & $\mathrm{L}$ & III & 1 & & & & \\
\hline \multirow{2}{*}{14} & \multirow{2}{*}{43} & \multirow{2}{*}{$\mathrm{F}$} & & $\mathbf{R}$ & & & II & 2 & & \\
\hline & & & & $\mathrm{L}$ & III & 2 & & & & \\
\hline \multirow{2}{*}{15} & \multirow{2}{*}{66} & $E_{F}$ & & $\mathrm{R}$ & III & 1 & & & & \\
\hline & & $F$ & & $\mathrm{~L}$ & III & 1 & & & & \\
\hline & & & & $\mathbf{R}$ & III & 2 & & & & \\
\hline 16 & 64 & $\mathrm{~F}$ & & $\mathrm{~L}$ & & & & & & \\
\hline 17 & 79 & $F_{F}$ & & $R$ & & & & & I & 1 \\
\hline 17 & 79 & $\mathrm{r}$ & & $\mathrm{L}$ & III & 2 & & & I & 1 \\
\hline 18 & 59 & $\mathrm{~F}$ & & $R$ & III & 11 & & & IV & 3 \\
\hline 18 & 59 & $r$ & & $\mathrm{~L}$ & & & & & $\mathrm{I}$ & 1 \\
\hline 19 & 66 & $\mathrm{~F}$ & & $R$ & III & 2 & & & I & 1 \\
\hline 19 & 66 & $F$ & & $\mathrm{~L}$ & III & 4 & & & & \\
\hline 20 & 73 & $F$ & 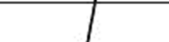 & $\mathbf{R}$ & III & 2 & & & & \\
\hline 20 & 73 & $F$ & & $\mathrm{~L}$ & III & 2 & & & & \\
\hline & & $ᄃ$ & 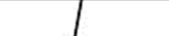 & $\mathbf{R}$ & III & 1 & & & & \\
\hline 21 & 84 & $\mathrm{~F}$ & & $\mathrm{~L}$ & III & 1 & & & IV & 3 \\
\hline 22 & 38 & $\mathrm{~F}$ & & $R$ & III & 6 & I & 2 & & \\
\hline & & & & $\mathrm{L}$ & III & 3 & & & & \\
\hline 23 & 62 & $F$ & & $\mathrm{R}$ & III & 4 & & & & \\
\hline 23 & 62 & $F$ & & L & III & 4 & & & I & 1 \\
\hline 24 & 77 & $F$ & & $\mathrm{R}$ & III & 4 & & & & \\
\hline 24 & $\pi$ & $F$ & & $\mathrm{~L}$ & III & 8 & & & & \\
\hline 25 & 33 & 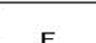 & & $\mathrm{R}$ & III & 7 & & & & \\
\hline 25 & 33 & $\mathrm{~F}$ & & $\mathrm{~L}$ & III & 2 & & & & \\
\hline 26 & 62 & $F$ & & $\mathbf{R}$ & III & 1 & & & & \\
\hline 20 & 02 & & & $\mathrm{~L}$ & III & 1 & II & 1 & & \\
\hline 27 & 64 & $F$ & & $\mathbf{R}$ & III & 1 & & & III & 2 \\
\hline 27 & 64 & $\mathrm{~F}$ & & $\mathrm{~L}$ & III & 1 & & & III & 2 \\
\hline 0 & 50 & $E$ & Fthonent & $R$ & III & 1 & & & & \\
\hline 28 & 59 & $F$ & Etanercept & L & III & 1 & & & & \\
\hline 29 & 69 & $\mathrm{~F}$ & Etanercent & $R$ & III & 15 & & & I & 4 \\
\hline 29 & 69 & $\mathrm{~F}$ & Etanercept & $\mathrm{L}$ & III & 11 & & & I & 1 \\
\hline 30 & 65 & $M$ & Etanercept & $\mathbf{R}$ & III & 6 & & & III & 3 \\
\hline 30 & 65 & $M$ & Golimumab & $\mathrm{L}$ & III & 1 & & & IV & 4 \\
\hline 31 & 75 & $\mathrm{~F}$ & Etanercept & $R$ & III & 8 & & & IV & 1 \\
\hline 31 & 15 & $r$ & Tocilizumab & $\mathrm{L}$ & III & 2 & & & & \\
\hline 30 & & $E$ & Adalimumab & $R$ & III & 2 & & & & \\
\hline 32 & 75 & $F$ & Adalimumab & L & III & 1 & & & & \\
\hline 33 & 65 & $\mathrm{~F}$ & Infliximab & $R$ & III & 3 & II & 3 & & \\
\hline 33 & 65 & $F$ & Tocilizumab & L & III & 2 & I & 3 & & \\
\hline
\end{tabular}

Figure 3. Number of injections in grade III patients. Thirty-three out of 118 patients were Grade III. 


\begin{tabular}{|c|c|c|c|c|c|c|c|c|c|c|}
\hline \multirow{2}{*}{ No. } & \multirow{2}{*}{ age } & \multirow{2}{*}{$\operatorname{sex}$} & \multirow{2}{*}{ Bio. } & \multirow{2}{*}{ site } & \multicolumn{2}{|c|}{ Wrist } & \multicolumn{2}{|c|}{ Elbow } & \multicolumn{2}{|c|}{ Shoulder } \\
\hline & & & & & grade & $\begin{array}{l}\text { injection } \\
\text { time }\end{array}$ & grade & $\begin{array}{l}\text { injection } \\
\text { time }\end{array}$ & grade & $\begin{array}{l}\text { injection } \\
\text { time }\end{array}$ \\
\hline 1 & 78 & $\mathrm{~F}$ & & $R$ & IV & 2 & & & & \\
\hline 2 & 80 & $\mathrm{~F}$ & & L & IV & 1 & & & & \\
\hline 3 & 75 & $\mathrm{~F}$ & & $\mathbf{R}$ & IV & 2 & & & & \\
\hline 4 & 50 & $\mathrm{~F}$ & & L & IV & 2 & & & & \\
\hline 5 & 71 & $\mathrm{~F}$ & & $\mathbf{R}$ & IV & 1 & & & & \\
\hline 6 & 68 & $\mathrm{~F}$ & & L & IV & 2 & IV & 3 & & \\
\hline 7 & 79 & $\mathrm{~F}$ & Tocilizumab & L & IV & 2 & & & & \\
\hline 8 & 56 & $\mathrm{~F}$ & Infliximab & L & IV & 2 & & & & \\
\hline 9 & 70 & $\mathrm{~F}$ & $\begin{array}{l}\text { Etanercept } \\
\text { Abatacept }\end{array}$ & $\mathbf{R}$ & IV & 2 & & & I & 5 \\
\hline \multirow{2}{*}{10} & \multirow{2}{*}{45} & \multirow{2}{*}{$\mathrm{F}$} & & $\mathbf{R}$ & IV & 13 & III & 10 & & \\
\hline & & & & $\mathrm{L}$ & IV & 19 & I & 2 & III & 2 \\
\hline \multirow{2}{*}{11} & \multirow{2}{*}{58} & \multirow{2}{*}{$\mathrm{F}$} & & $\mathbf{R}$ & IV & 6 & & & & \\
\hline & & & & L & IV & 5 & & & & \\
\hline \multirow{2}{*}{12} & \multirow{2}{*}{62} & \multirow{2}{*}{$\mathrm{F}$} & & $\mathbf{R}$ & IV & 3 & & & & \\
\hline & & & & L & IV & 4 & I & 1 & & \\
\hline \multirow{2}{*}{13} & \multirow{2}{*}{83} & \multirow{2}{*}{$\mathrm{F}$} & & $\mathbf{R}$ & IV & 11 & & & & \\
\hline & & & & L & IV & 9 & & & & \\
\hline \multirow{2}{*}{14} & \multirow{2}{*}{75} & \multirow{2}{*}{ M } & & $\mathbf{R}$ & IV & 2 & & & & \\
\hline & & & & L & IV & 5 & & & & \\
\hline 15 & 70 & $\mathrm{M}^{2}>$ & & $\mathbf{R}$ & IV & 2 & I & 2 & & \\
\hline & r & 101 & & L & IV & 1 & & & & \\
\hline 16 & 60 & $\mathrm{~F}$ & & $\mathbf{R}$ & IV & 1 & & & & \\
\hline & & & & L & IV & 1 & & & & \\
\hline 17 & Oم & $F$ & Colimumoul & $\mathbf{R}$ & IV & 2 & & & & \\
\hline 17 & 00 & 1 & 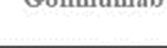 & L & IV & 1 & & & & \\
\hline 18 & 63 & $F_{2}$ & Ftanercent & $\mathbf{R}$ & IV & 2 & I & 4 & I & 1 \\
\hline & & & & L & & & I & 1 & & \\
\hline 10 & 10 & 5 & Yalimumol & $\mathbf{R}$ & IV & 2 & & & & \\
\hline 10 & 70 & 1 & 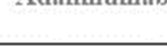 & L & II & 1 & & & & \\
\hline 20 & 84 & $F$ & Ftanercent & $\mathbf{R}$ & IV & 1 & & & & \\
\hline 20 & 04 & $\Gamma$ & Ltanercept & L & IV & 1 & & & & \\
\hline 21 & 17 & $E$ & Tnflivimb & $\mathbf{R}$ & IV & 12 & & & & \\
\hline 21 & 41 & 1 & 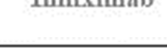 & L & IV & 12 & & & & \\
\hline 22 & 71 & $F$ & thatacent & $\mathbf{R}$ & IV & 2 & & & & \\
\hline & & & Aonatapept & L & IV & 2 & & & & \\
\hline 23 & 63 & 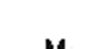 & Etanercept & $\mathbf{R}$ & IV & 5 & & & & \\
\hline 25 & 03 & $m$ & Abatacept & $\mathrm{L}$ & IV & 5 & & & & \\
\hline
\end{tabular}

Figure 4. Number of injections in grade IV patients. Twenty-three out of 118 patients were Grade IV. 


\begin{tabular}{|c|c|c|c|c|c|c|c|c|}
\hline \multicolumn{5}{|c|}{ except wrist joint } & \multicolumn{2}{|c|}{ Elbow } & \multicolumn{2}{|c|}{ Shoulder } \\
\hline No. & age & sex & Biologi & site & grade & $\begin{array}{c}\text { injection } \\
\text { time }\end{array}$ & grade & $\begin{array}{c}\text { injection } \\
\text { time }\end{array}$ \\
\hline 1 & 70 & $\mathrm{~F}$ & & $\mathrm{R}$ & & & IV & 10 \\
\hline 2 & 82 & $M$ & & $\mathbf{R}$ & & & IV & 6 \\
\hline 3 & 53 & $\mathrm{~F}$ & & $\mathbf{R}$ & I & 1 & & \\
\hline 4 & 69 & $\mathrm{~F}$ & & $\mathbf{R}$ & III & 1 & & \\
\hline 5 & 67 & $F$ & & $\mathbf{R}$ & & & IV & 2 \\
\hline 6 & 38 & $\mathrm{~F}$ & & $\mathrm{~L}$ & & & & \\
\hline 7 & 43 & $\mathrm{~F}$ & & $\mathbf{R}$ & I & 2 & & \\
\hline 8 & 51 & $\mathrm{~F}$ & & & I & 1 & I & 1 \\
\hline 9 & 66 & $\mathrm{~F}$ & & $\mathrm{~L}$ & & & I & 1 \\
\hline 10 & 86 & $\mathrm{~F}$ & & $\mathrm{~L}$ & & & I & 1 \\
\hline 11 & 73 & $\mathrm{~F}$ & & $\mathrm{~L}$ & & & I & 3 \\
\hline 12 & 64 & $\mathrm{~F}$ & & $\mathbf{R}$ & & & I & 2 \\
\hline 13 & 64 & $\mathrm{~F}$ & Infliximab & $\mathbf{R}$ & IV & 8 & & \\
\hline 14 & 72 & $M$ & Infliximab & $\mathrm{L}$ & II & 2 & III & 2 \\
\hline \multirow{2}{*}{17} & \multirow{2}{*}{78} & \multirow{2}{*}{$F$} & & $\mathrm{R}$ & I & 1 & & \\
\hline & & & & L & I & 2 & & \\
\hline \multirow{2}{*}{18} & \multirow{2}{*}{85} & \multirow{2}{*}{$M$} & & $\mathbf{R}$ & I & 1 & & \\
\hline & & & & $\mathrm{L}$ & I & 2 & & \\
\hline \multirow{2}{*}{19} & \multirow{2}{*}{46} & \multirow{2}{*}{$F$} & & $\mathbf{R}$ & & & I & 1 \\
\hline & & & & $\mathrm{L}$ & II & 1 & & 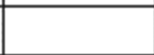 \\
\hline \multirow{2}{*}{20} & \multirow{2}{*}{59} & \multirow{2}{*}{$\mathrm{F}$} & & $\mathrm{R}$ & III & 2 & IV & 1 \\
\hline & & & & $\mathrm{L}$ & & & IV & 17 \\
\hline \multirow{2}{*}{21} & \multirow{2}{*}{67} & \multirow{2}{*}{$\mathrm{F}$} & & $\mathbf{R}$ & & & III & 2 \\
\hline & & & & $\mathrm{L}$ & & & II & 3 \\
\hline \multirow{2}{*}{22} & \multirow{2}{*}{79} & \multirow{2}{*}{$\mathrm{F}$} & & $\mathrm{R}$ & I & 1 & & \\
\hline & & & & $\mathrm{L}$ & II & 1 & & \\
\hline \multirow{2}{*}{25} & \multirow{2}{*}{52} & & & $\mathrm{R}$ & & & I & 2 \\
\hline & & $\mathrm{r}$ & & L & & & I & 2 \\
\hline & & & & $\mathrm{R}$ & & & I & 14 \\
\hline 27 & 12 & $M$ & & $\mathrm{~L}$ & & & I & 14 \\
\hline & & & & $\mathbf{R}$ & IV & 2 & & \\
\hline 28 & 68 & $F$ & & $\mathrm{~L}$ & IV & 5 & & \\
\hline 20 & 53 & E & Shatacont & $R$ & I & 1 & & \\
\hline 29 & 53 & $r$ & ADatacept & $\mathrm{L}$ & I & 1 & & \\
\hline & & & & $\mathbf{R}$ & & & IV & 3 \\
\hline 30 & 69 & $F$ & Etanercept & $\mathrm{L}$ & & & IV & 3 \\
\hline 31 & 66 & $\mathrm{E}$ & Infliximah & $\mathrm{R}$ & IV & 5 & & \\
\hline 31 & 60 & $r$ & Infliximab & $\mathrm{L}$ & & & IV & 5 \\
\hline & & & Infliximab, & $\mathbf{R}$ & & & I & 1 \\
\hline 32 & 12 & $M$ & Adalimumab & $\mathrm{L}$ & III & 2 & & \\
\hline & & & & $\mathrm{R}$ & & & IV & 1 \\
\hline 33 & 72 & $\mathrm{~F}$ & Adalimumab & $\mathrm{L}$ & III & 1 & IV & 10 \\
\hline
\end{tabular}

Figure 5. Number of injections in except the wrist joint. Thirty-three out of 118 patients were injected elbow and shoulder joint. 


\section{Results}

\subsection{Number of Injections}

The mean numbers of injections were as follows: wrist, grade I, 3.4; grade II, 4.1; grade III, 3.1; grade IV, 4.1; overall, 3.7; elbow, grade I, 1.7; grade II, 1.6; grade III, 2.6; grade IV, 4.6; overall, 2.2; shoulder, grade I, 2.2; grade II, 2.0; grade III, 1.6; grade IV, 5.0; overall, 2.8.

Five females with 5 wrist joints (Grade II: 4 females, Grade IV: 1 female) and 2 men with 2 wrists (Grade III) underwent Sauvè-Kapandji. Extensor tendon rupture occurred in 2 females. One of these patients (grade II) was underwent Sauvé-Kapandji procedure and tendon transplantation were performed. The other (grade IV) refused surgery and was kept under observation; however, the extensor disorder of her fingers seemed to be limited and she experienced no inconvenience in her daily life. Three females out of 5 females wanted to operate before injection, but for the convenience of home, injection was performed until the operation. Two men (grade III) had an injection effect but were in heavy labor, so they wanted surgery and performed Sauvé-Kapandji procedure. Therefore, we removed those 7 cases from the total number.

\subsection{Use of Biologics}

The overall average value to the wrist, elbow and shoulder were 3.7, 2.2 and 2.8 (Table 6). For the patients who did not receive a biologic, the mean numbers of injections to the wrist, elbow and shoulder were 3.4, 1.9 and 2.4, respectively, whereas the mean numbers for those who received biologics were 3.3, 2.3 and 2.3, respectively. There was no remarkable difference in the numbers of injections between the patients who received and who did not receive biologics (Table 7).

\begin{tabular}{|c|c|c|c|c|}
\hline wrist & case & $\begin{array}{c}\text { total } \\
\text { wrist } \\
\text { no. }\end{array}$ & $\begin{array}{c}\text { total inj. } \\
\text { times }\end{array}$ & average \\
\hline Grade I & 32 & 48 & 165 & 3.4 \\
\hline Grade II & 30 & 46 & 193 & 4.1 \\
\hline Grade III & 33 & 51 & 158 & 3.1 \\
\hline Grade IV & 23 & 35 & 145 & 4.1 \\
\hline total & 118 & 180 & 661 & 3.7 \\
\hline
\end{tabular}

\begin{tabular}{|c|c|c|c|c|}
\hline elbow & case & $\begin{array}{c}\text { total } \\
\text { elbow } \\
\text { no. }\end{array}$ & $\begin{array}{c}\text { total inj. } \\
\text { times }\end{array}$ & average \\
\hline Grade I & 17 & 24 & 41 & 1.7 \\
\hline Grade II & 7 & 7 & 11 & 1.6 \\
\hline Grade III & 8 & 9 & 23 & 2.6 \\
\hline Grade IV & 4 & 5 & 23 & 4.6 \\
\hline total & 36 & 45 & 98 & 2.2 \\
\hline
\end{tabular}

\begin{tabular}{|c|c|c|c|c|}
\hline shoulder & case & $\begin{array}{c}\text { total } \\
\text { shoulder } \\
\text { no. }\end{array}$ & $\begin{array}{c}\text { total inj. } \\
\text { times }\end{array}$ & average \\
\hline Grade I & 25 & 37 & 82 & 2.2 \\
\hline Grade II & 2 & 2 & 4 & 2 \\
\hline Grade III & 6 & 7 & 11 & 1.6 \\
\hline Grade IV & 11 & 14 & 70 & 5 \\
\hline total & 44 & 60 & 167 & 2.8 \\
\hline
\end{tabular}

Figure 6. Number of injections. The overall average value to the wrist, elbow and shoulder were 3.7, 2.2 and 2.8.

\begin{tabular}{|c|c|c|c|}
\cline { 2 - 4 } \multicolumn{1}{c|}{} & \multicolumn{3}{c|}{ Bio. Unsed } \\
\hline inj. Times & wrist & elbow & shoulder \\
\hline cases & 478 & 70 & 109 \\
\hline inj. Times (average) & 139 & 36 & 45 \\
\hline & 3.4 & 1.9 & 2.4 \\
\cline { 2 - 4 }
\end{tabular}

\begin{tabular}{|c|c|c|c|}
\cline { 2 - 4 } \multicolumn{1}{c|}{} & \multicolumn{3}{c|}{ Bio. Used } \\
\hline inj. Times & wrist & elbow & shoulder \\
\hline cases & 264 & 36 & 63 \\
\hline inj. Times (average) & 81 & 16 & 27 \\
\hline & 3.3 & 2.3 & 2.3 \\
\cline { 2 - 4 }
\end{tabular}

Figure 7. Injection times by use Biologic or not. For the patients who did not receive a biologic, the mean numbers of injections to the wrist, elbow and shoulder were 3.4, 1.9 and 2.4. The mean numbers for those who received biologics were 3.3, 2.3 and 2.3.

\subsection{Decrease in Pain}

Mean improvements in VAS pain scores from baseline were as follows: wrist, from 75 to $11 \mathrm{~mm}$; elbow, from 79 to $17 \mathrm{~mm}$; shoulder, from 54 to $11 \mathrm{~mm}$.

\subsection{Adverse Effects of Intra-articular Triamcinolone Acetonide Injections}

No abnormalities were observed in subcutaneous tissue and the extensor tendon.

\subsection{Changes in CHS, RCDR and RRA with and Without Injections of Triamcinolone Acetonide}

Table 8 summarizes the analysis of changes over a mean follow-up period of 8 years for the 2 groups of patients with grade III and IV wrists who received and did not receive intra-articular injections of triamcinolone acetonide. The group that received the injections showed no significant changes in CHR (from $0.423 \pm 0.0714$ to $0.413 \pm 0.0919$ ) or RCDR from $0.356 \pm 0.0571$ at baseline to $0.354 \pm 0.0658$, whereas RRA changed significantly decrease (from $111.2 \pm 10.577$ degrees to $107.5 \pm 9.2$ degrees $)(\mathrm{P}<0.05)$. In the group that did not receive intra-articular injections, no significant changes were observed in CHR (from $0.441 \pm 0.0791$ to $0.443 \pm 0.0717$ ) or RCDR (from $0.354 \pm 0.0661$ at baseline to $0.358 \pm 0.0435$ ), or RRA (from $109.8 \pm 10.426$ degrees to $136.2 \pm 15.9$ degrees). 


\begin{tabular}{|c|c|c|c|}
\hline Triamcinolone Acetonide injected & Pre-inj. & Post-inj. & p value \\
\hline \multicolumn{4}{|l|}{ (Grade III N=22) } \\
\hline Carpal height ratio (CTS) & $0.407 \pm 0.0693$ & $0.407 \pm 0.0942$ & NS \\
\hline Radiocarpal distance ratio (RCDR) & $0.359 \pm 0.0630$ & $0.352 \pm 0.0704$ & NS \\
\hline Radial rotation angle (RRA) & $112.7 \pm 12.1$ & $107.5 \pm 10.1$ & $P<0.05$ \\
\hline \multicolumn{4}{|l|}{ (Grade IV N=15) } \\
\hline Carpal height ratio (CTS) & $0.447 \pm 0.0703$ & $0.422 \pm 0.0908$ & NS \\
\hline Radiocarpal distance ratio (RCDR) & $0.35 \quad \pm \quad 0.0488$ & $0.36 \pm 0.0603$ & NS \\
\hline Radial rotation angle (RRA) & $108.9 \pm 7.666$ & $107.6 \pm 8.1$ & NS \\
\hline \multicolumn{4}{|l|}{ (Grade III $+\mathrm{IV} \mathrm{N}=37$ ) } \\
\hline Carpal height ratio (CTS) & $0.423 \pm 0.0714$ & $0.413 \pm 0.0919$ & NS \\
\hline Radiocarpal distance ratio (RCDR) & $0.356 \pm 0.0571$ & $0.354 \pm 0.0658$ & NS \\
\hline Radial rotation angle (RRA) & $111.2 \pm 10.577$ & $107.5 \pm 9.2$ & $P<0.05$ \\
\hline
\end{tabular}

\begin{tabular}{|c|c|c|c|}
\hline Triamcinolone Acetonide non-injected & Pre-inj. & Post-inj. & p value \\
\hline \multicolumn{4}{|l|}{\begin{tabular}{|c|c} 
& (Grade III $N=16)$ \\
\end{tabular}} \\
\hline Carpal height ratio (CTS) & $0.463 \pm 0.0768$ & $0.468 \pm 0.0590$ & NS \\
\hline Radiocarpal distance ratio (RCDR) & $0.352 \pm 0.0361$ & $0.345 \pm 0.0491$ & NS \\
\hline Radial rotation angle (RRA) & $114.7 \pm 9.5129$ & $117.6 \pm 9.1$ & NS \\
\hline
\end{tabular}

\begin{tabular}{|c|c|c|c|}
\hline \multicolumn{4}{|l|}{ (Grade IV $N=17$ ) } \\
\hline Carpal height ratio (CTS) & $0.42 \pm 0.0778$ & $0.419 \pm 0.076$ & NS \\
\hline Radiocarpal distance ratio (RCDR) & $0.355 \pm 0.0867$ & $0.37 \pm 0.0346$ & NS \\
\hline Radial rotation angle (RRA) & $105.1 \pm 9.2187$ & $112.3 \pm 8.3$ & $P<0.05$ \\
\hline
\end{tabular}

\begin{tabular}{|c|c|c|c|}
\hline \multicolumn{4}{|l|}{ (Grade III+IV N=33) } \\
\hline Carpal height ratio (CTS) & $0.441 \pm 0.0791$ & $0.443 \pm 0.0717$ & NS \\
\hline Radiocarpal distance ratio (RCDR) & $0.354 \pm 0.0661$ & $0.358 \pm 0.0435$ & NS \\
\hline Radial rotation angle (RRA) & $109.8 \pm 10.416$ & $136.2 \pm 15.9$ & NS \\
\hline
\end{tabular}

Figure 8. Comparison of CHR, RCDR, and RRA as determined by dorso-palmar plain X-ray imaging in grade III/IV patients. The group that received the injections showed no significant changes in CHR or RCDR, whereas RRA changed significantly decrease $(P<0.05)$. In the group that did not receive intra-articular injections, no significant changes were observed in CHR or RCDR, or RRA.

\section{Discussion}

Recently, biologic agents have been used successfully for the control of RA. Nevertheless, many patients with a long history of RA complain of wrist pain. The Sauvé-Kapandji procedure is our first recommendation for RA patients with persistent wrist pain of at least Larsen grade II. This surgical method improved wrist pain and range of motion after surgery. Many patients with RA complain of wrist pain despite the use of biologic therapy. At our hospital, we treat those who refuse surgery with a unilateral injection of $20 \mathrm{mg}$ of triamcinolone acetonide and $5 \mathrm{~mL}$ of $1 \%$ lidocaine hydrochloride into the joints of RA to alleviate persistent pain. We have previously reported the efficacy and safety of this intra-articular therapy for wrist pain [3]. In the present study, we evaluated the effects of injections of triamcinolone acetonide into the wrist, elbow and shoulder joints.

The mean number of injections per patient for wrist joints was 3.7. The mean number of injections into elbow joints was slightly higher (4.6) for grade IV elbows, although the overall mean was 2.2. The mean number of injections into shoulder joints was even higher (5.0) for grade IV shoulders, with an overall mean of 2.8 . In all the joints evaluated, the number of injections increased as the grade increased; however, the overall mean number of injections was $\leq 4$, indicating the benefit of the injections. Whether or not biologics were used, the number of injections depended on the location and grade; 
in both groups, the mean number of injections frequency was $\leq 4$, with no significant difference between the groups. The reason for this was that the use of biologics for patients with grade III and IV joints suppressed the proliferation of the synovial membrane but the pain due to joint destruction remained, requiring an intra-articular injection of triamcinolone acetonide to suppress this pain. The patients who did not receive biologics experienced joint destruction, but the pathology of RA was controlled, and pain due to the joint destruction could be tolerated. The observation that joint destruction did not proceed after a triamcinolone acetonide injection during 8 years in X-P, and it was probably due to suppression of synovial proliferation by the injection.

Improvements in pain VAS scores were observed in all joints, which may have been the result of the injections.

Using simple X-ray front views before and after the injection, we investigated whether the intra-articular injection resulted in any progression of the wrist bone destruction. However, our analysis of the grade III, grade IV, and grade III + IV groups of patients with wrist pain available for follow-up, no significant differences in CHR or RCDR were observed between those who received triamcinolone acetonide injections and those who did not. However, RRA decreased significantly in the patients who received the injections.

Many investigators have studied intra-articular corticosteroid injections. Chandler et al. [4] Noted substantial pain relief from intra-articular knee injections of hydrocortisone. Subsequent loading to the knee may have conferred an excessive burden on the knee unconsciously and resulted in joint damage in some patients. In the 1970s, clinical investigations by Salter et al. [5] And Gray et al. [6] Identified no direct relationship between the number of corticosteroid injections into the knee and joint destruction. We investigated whether joint destruction progressed after injecting triamcinolone acetonide into the wrist, elbow, and shoulder joints, which are not weight-bearing joint. The mean number of injections was 3.7, 2.2, 2.8 and no remarkable progression of joint destruction was observed. Ostergaard et al. [7] Noted that intra-articular triamcinolone acetonide conferred a longer response than other corticosteroids and recommended up to 3 to 4 injections per year, with a 6 -week dosing interval. In the present study, the mean number of injections to all joints within the 8-year study period was $<4$. It is therefore difficult to determine whether changes were due to the progression of RA or to side effects of the triamcinolone acetonide. However, the lack of any acute joint destruction following the injection suggests that triamcinolone acetonide was not responsible.

In the early $2000 \mathrm{~s}$, there were many reports from Western institutions of treating RA patients with wrist injections of corticosteroids [8, 9]. Koski et al. [10] Reported that $68 \%$ of patients who received corticosteroids for the wrists maintained a clinically normal condition for 3 months after treatment, with 20\% of the patients showing synovia with a normal appearance on ultrasonography, suggesting that corticosteroids suppressed the proliferation of the synovial membrane in the joint. Haugeberg et al. [11] Evaluated injections of corticosteroids into the wrists and metacarpal joints of patients with early-stage RA and concluded that corticosteroid treatment may improve RA-induced joint degradation after 3 months. Hetland et al. [12, 13] Reported that injections of corticosteroids into the joints of the hands, elbows, shoulders, knees, and feet in early-stage RA patients resulted in the 2-year effective control of RA and probable preservation of better joint function compared with bone destruction due to RA.

Intra-articular injections with glucocorticoids are reported standard procedures according to therapy guidelines in many rheumatic conditions [14, 15]. As another reports of using steroids and Biologic in combination have also been found. Axelsen et al. [16] Reported that adalimumab was injected into the wrist joint, effectively reducing synovitis, osteomyelitis, and tendon synovitis, and stopping the progression of structural damage as judged by MRI. Since then, biologics have generally infusion or subcutaneous injection has become the main treatment. Kanbe et al. [17] Reported simultaneous treatment with biologics and intra-articular injection of Triamcinolone Acetonide is useful for cases involving switching of biologics for RA. This strategy is safe and practical for RA treatment. Hørslev-Petersen $K$ et al. [18] Reported an aggressive triamcinolone and synthetic DMARD treat-to-target strategy in early RA provided excellent 2-year clinical and radiographic disease control independent of adalimumab induction therapy.

In a recent study on 228 patients with knee osteoarthritis, Bodick et al. [19] Reported that sustained-release triamcinolone acetonide improved pain relief effect better than the currently used immediate-release triamcinolone acetonide. Kumar et al. [20] Reported similar results in a study using rats. In many cases, the number of injections decreased as the number of infusions increased over time. In 2018, the extended-release formulation of triamcinolone acetonide has been reported to be more effective than the currently used triamcinolone acetonide for osteoarthritis of the knee [21-23].

Steroid arthropathy can result from the injection of steroids, and the intra-articular injection method is not widely used in Japan. In addition, there have been reports of problems with triamcinolone acetonide in hand surgery for non-RA patients, including tendon rupture in a trigger finger [24] and post-injection subcutaneous atrophy and pigmentation loss in patients with DeQuervain's tenosynovitis and lateral epicondylitis; these findings highlight the need for caution.

There have been no previous Japanese reports describing long-term follow-up investigations of patients receiving intra-articular triamcinolone acetonide injections for joint pain. The wrist, elbow, and shoulder are not weight-bearing, and intra-articular injections of triamcinolone acetonide at $20 \mathrm{mg}$ are sufficient to relieve joint pain. Although many of our patients achieved a long-term response with a single injection during the treatment period. Skin atrophy or extensor tendon rupture due to injection did not occur because the drugs were appropriately injected into the joints. In our examination, 49 of 153 cases complained of joint pain despite using biologics. The effect of intra-articular injection of triamcinolone acetonide depends on the grade, but the average number of injections was 4 or less regardless of whether or not biologics were administered. Intra-articular triamcinolone acetonide does not stop RA progression but offers an option for relieving joint pain. 


\section{Conclusion}

To treat RA patients with persistent wrist, elbow and shoulder pain, and $20 \mathrm{mg}$ of triamcinolone acetonide and 5 $\mathrm{mL}$ of $1 \%$ lidocaine hydrochloride were injected into the joint. An additional $25 \mathrm{mg}$ of hyaluronic acid was added into the shoulder joint. The mean number of injections per patient over the study period of 8 years was $<4$. There was no difference in the number of injections between the patients who received biologics and those who did not. No subcutaneous atrophy resulted from the injections. Extensor tendon rupture occurred in 2 cases but the injection was not considered to be a direct cause.

\section{Appendix}

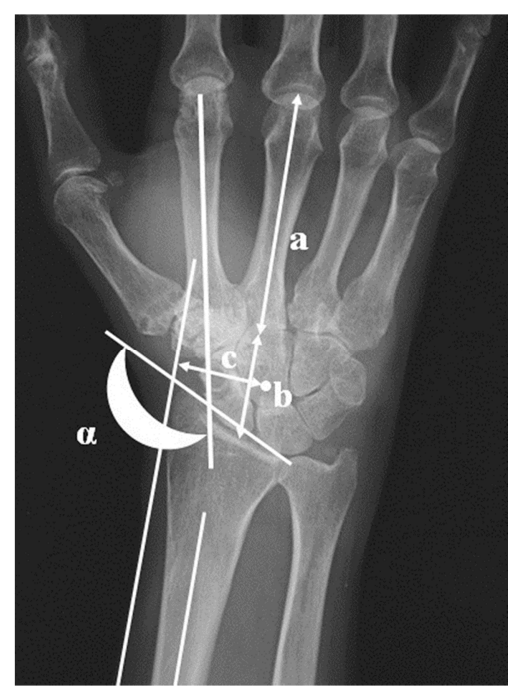

Figure 9. Methods of measuring carpal height, radio-carpal distance and radial rotation angle of the carpus.

\section{References}

[1] Larsen A, Dale K, Eek M. Radiographic evaluation of rheumatoid arthritis and related conditions by standard reference films. Acta Radio Diagn. 1977; 18 (49), 481-91.

[2] Youm Y, McMurthy RY, Flatt AE, Gillespie TE. Kinematics of the wrist. I. An experimental study of radial-ulnar deviation and flexion-extension. J Bone Joint Surg Am; 1978; 60 (4): 423-31.

[3] Akihiro Fukui, Hideki Yamada, Takashi Yoshii. Effect of Intraarticular Triamcinolone Acetonide injection for Wrist Pain in Rheumatoid Arthritis Patients. J Hand Surg Asian Pac. 2016; 21 (2): 239-45.

[4] Chandler GN, Wright V. Deleterious effect of intra-articular hydrocortisone. Lancet. 1958; 2 (7048): 661-3.

[5] Salter RB, Gross A, Hall JH. Hydrocortisone arthropathy- an experimental investigation. Can Med Assoc J. 1967; 19; 97 (8): 374-7.

[6] Gray RG, Gottlieb NL. Intra-articular corticosteroids. An updated assessment. Clin Orthop Relat Res. 1983; (177): 235-63.
[7] Ostergaard M, Halberg P. Intra-articular glucocorticoid injections in joint diseases. Ugeskr Laeger. 1999; 1: 161 (5): 582-6.

[8] Furtado RN, Oliveira LM, Natour J. Polyarticular corticosteroid injection versus systemic administration in treatment of rheumatoid arthritis patients: a randomized controlled study, J Rheumatol. 2005; 32 (9): 1691-8.

[9] Lopes RV, Furtado RN, Parmigiani L, Rosenfeld A, Fermandes AR, Natour J. Accuracy of intra-articular injections in peripheral joints performed blindly in patients with rheumatoid arthritis. Rheumatology (Oxford). 2008; 47 (12); 1792-4.

[10] Koski JM, Hermunen H. Intra-articular glucocorticoid treatment of the rheumatoid wrist. An ultrasonographic study. Scand J Rheumatol. 2001; 30 (5): 268-70.

[11] Haugeberg G, Morton S, Emery P, Conaghan PG. Effect of intra-articular corticosteroid injections and inflammation on periarticular and generalized bone loss in early rheumatoid arthritis. Ann Rheum Dis. 2011; 70 (1): 184-7.

[12] Hetland ML, Stengaard-Pedersen K, Junker P, Lottenburger T, Hansen I, Andersen LS, Tarp U, Svendsen A, Pedersen JK, Skjødt H, Lauridsen UB, Ellingsen T, Hansen GV, Lindegaard H, Vestergaard A, Jurik AG, Østergaard M, Hørslev-Petersen $\mathrm{K}$; CIMESTRA study group.. Aggressive combination therapy with intra-articular glucocorticoid injections and conventional disease-modifying anti-rheumatic drugs in early rheumatoid arthritis: second-year clinical and radiographic results from the CIMESTRA study. Ann Rheum Dis. 2008; 67 (6): 815-22.

[13] Hetland ML, Østergaard M, Ejbjerg B, Jacobsen S, Stengaard-Pedersen K, Junker P, Lottenburger T, Hansen I, Andersen LS, Tarp U, Svendsen A, Pedersen JK, Skjødt H, Ellingsen T, Lindegaard H, Pødenphant J, Hørslev-Petersen K, CIMESTRA study group. Short- and long-term efficacy of intra-articular injections with betamethasone as part of a treat-to-target strategy in early rheumatoid arthritis: impact of joint area, repeated injections, MRI findings, anti-CCP, IgM-RF and CRP. Ann Rheum Dis. 2012; 71 (6): 851-6.

[14] Menon N, Kothari SY, Gogna A, Sharma R. Comparison of intra-articular glucocorticoid injections with DMARDs versus DMARDs alone in rheumatoid arthritis. J Assoc Physicians India. 2014; 62 (8): 673-6.

[15] Hammer M, Schwarz T, Ganser G. Intra-articular injection of cortisone. Z Rheumatol. 2015; 74 (9): 774-9.

[16] Axelsen MB, Eshed I, Hørslev-Petersen K, Stengaard-Pedersen K, Hetland ML, Møller J, Junker P, Pødenphant J, Schlemmer A, Ellingsen T, Ahlquist P, Lindegaard H, Linauskas A, Dam MY, Hansen I, Horn HC, Ammitzbøll CG, Jørgensen A, Krintel SB, Raun J, Krogh NS, Johansen JS, Østergaard M; OPERA study group. A treat-to-target strategy with methotrexate and intra-articular triamcinolone with or without adalimumab effectively reduces MRI synovitis, osteitis and tenosynovitis and halts structural damage progression in early rheumatoid arthritis: results from the OPERA randomised controlled trial. Ann Rheum. 2015; 74 (5): 867-75.

[17] Kanbe K, Chiba J, Inoue Y, Taguchi M, Yabuki A, Simultaneous Treatment with Subcutaneous Injection of Golimumab and Intra-articular Injection of Triamcinolone Acetonide (K-Method) in Patients with Rheumatoid Arthritis Undergoing Switching of Biologics: Retrospective Case-Control Study. Clin Med Insights Arthritis Musculoskelet Disord. 2016; 4 (9): 45-9. 
[18] Hørslev-Petersen K, Hetland ML, Ørnbjerg LM, Junker P, Pødenphant J, Ellingsen $\mathrm{T}$, Ahlquist $\mathrm{P}$, Lindegaard $\mathrm{H}$, Linauskas A, Schlemmer A, Dam MY, Hansen I, Lottenburger T, Ammitzbøll CG, Jørgensen A, Krintel SB, Raun J, Johansen JS, Østergaard M, Stengaard-Pedersen K; OPERA Study-Group. Clinical and radiographic outcome of a treat-to-target strategy using methotrexate and intra-articular glucocorticoids with or without adalimumab induction: a 2-year investigator-initiated, double-blinded, randomised, controlled trial (OPERA). Ann Rheum Dis. 2016; 75 (9): 1645-53.

[19] Bodick N, Lufkin J, Willwerth C, Kumar A, Bolognese J, Schoonmaker C, Ballal R, Hunter D, Clayman M. An intra-articular extended-release formulation of triamcinolone acetonide prolongs and amplifies analgesic effect in patients with osteoarthritis of the knee: a randomized clinical trial. J Bone Joint Surg Am. 2015; 97 (11): 877-88.

[20] Kumar A, Bendele AM, Blanks RC, Bodick N. Sustained efficacy of a single intra-articular dose of FX006 in a rat model of repeated localized knee arthritis. Osteoarthritis Cartilage. 2015; 23 (1): 151-60.

[21] Conaghan PG, Hunter DJ, Cohen SB, Kraus VB Berenbaum F, Lieberman JR, Jones DG, Spitzer A, I Jevsevar DS, Katz NP,
Burgess DJ, Lufkin J, Johnson JR, Bodick N; FX0062014-008 Participating Investigators. Effects of a Single Intra-Articular Injection of a Microsphere Formulation of Triamcinolone Acetonide on Knee Osteoarthritis Pain: A Double-Blinded, Randomized, Placebo-Controlled, Multinational Study. J Bone Joint Surg Am. 2018; 18; 100 (8): 666-677.

[22] Conaghan PG, Cohen SB, Berenbaum F, Lufkin J, Johnson JR, Bodick N: Brief Report: A Phase IIb Trial of a Novel Extended-Release Microsphere Formulation of Triamcinolone Acetonide for Intraarticular Injection in Knee Osteoarthritis. Arthritis Rheumatol. 2018; 70 (2): 204-211.

[23] Kraus VB, Conaghan PG, Aazami HA, Mehra P, Kivitz AJ, Lufkin J, Hauben J, Johnson JR, Bodick N.: Synovial and systemic pharmacokinetics (PK) of triamcinolone acetonide (TA) following intra-articular (IA) injection of an extended-release microsphere-based formulation (FX006) or standard crystalline suspension in patients with knee osteoarthritis (OA). Osteoarthritis Cartilage. 2018; 26 (1): 34-42.

[24] Fizgerald BT, Hofmeister EP, Fan RA, Thompson MA. Delayed flexor digitorum superficialis and profundus rupture in a trigger finger after a steroid injection: a case report. J Hand Surg Am; 2005; 30 (3): 479-82. 